Теорія Ймовір. та Матем. Статист. Вип. 81, 2010
Theor. Probability and Math. Statist. No. 81, 2010, Pages 59-70 S 0094-9000(2010)00810-6

Article electronically published on January 18, 2011

\title{
AN INEQUALITY FOR THE LÉVY DISTANCE BETWEEN TWO DISTRIBUTION FUNCTIONS AND ITS APPLICATIONS
}

UDC 519.21

\author{
K.-H. INDLEKOFER, O. I. KLESOV, AND J. G. STEINEBACH
}

\begin{abstract}
We prove a nonuniform bound for the deviation between two distribution functions expressed in terms of the Lévy distance. Applications of this bound to the global version of the central limit theorem are given and complete convergence is shown.
\end{abstract}

\section{INTRODUCTION}

Let $F$ and $G$ be two distribution functions. Then the Lévy distance $\mathcal{L}(F, G)$ between $F$ and $G$ is defined as

$$
\mathcal{L}(F, G)=\inf \mathbb{H},
$$

where

$$
\mathbb{H}=\{h: G(x-h)-h \leq F(x) \leq G(x+h)+h \text { for all } x\} .
$$

The Lévy distance is much less popular in probability theory than the uniform distance

$$
\Delta(F, G)=\sup _{x \in \mathbf{R}}|F(x)-G(x)| .
$$

The advantage of the Lévy distance becomes evident if one considers the weak convergence

$$
F_{n} \stackrel{w}{\longrightarrow} G, \quad n \rightarrow \infty,
$$

which is equivalent to $\mathcal{L}\left(F_{n}, G\right) \rightarrow 0(n \rightarrow \infty)$ (see, for example, 7]). If $G$ is continuous, then the weak convergence $F_{n} \stackrel{w}{\longrightarrow} G(n \rightarrow \infty)$ is also equivalent to $\Delta\left(F_{n}, G\right) \rightarrow 0$ $(n \rightarrow \infty)$; however, this property may fail if $G$ has discontinuities. We also recall that always

$$
\mathcal{L}(F, G) \leq \Delta(F, G) .
$$

Having weak convergence in mind, we compare the deviation $|F(x)-G(x)|$ between two distribution functions. The bound we obtain in Section 2 is nonuniform in $x$ and is expressed in terms of the Lévy distance $\mathcal{L}(F, G)$. Furthermore, we discuss some examples of applications of our bound. In Section 3, we obtain a generalized global version of the central limit theorem. Finally, Section 4 contains some results for the generalized complete convergence.

2010 Mathematics Subject Classification. Primary 60J05.

Key words and phrases. Lévy distance, global version of the central limit theorem, complete convergence.

Supported by a DFG grant. 


\section{A BOUND IN TERMS OF THE LÉVY DISTANCE}

In this section, we concentrate on the case of $G=\Phi$ where $\Phi$ is the standard $\mathcal{N}(0,1)$ Gaussian law.

Bounds for $|F(x)-\Phi(x)|$ expressed in terms of the uniform distance have been studied in many papers. The most popular is the case where $F$ corresponds to a sum of independent random variables. Research in this direction originated from the work of Esseen [5].

Kolodyazhny [1] extends the results of [5] by proving the following theorem.

Theorem A (Kolodyazhnyı). Let $F$ be an arbitrary distribution function and let $\Delta=$ $\Delta(F, \Phi)$. Let $p>0$ and assume that the moment of order $p$ exists for the distribution function $F$. Denote

$$
\lambda_{p}=\left.\left|\int_{-\infty}^{\infty}\right| x\right|^{p} d F(x)-\int_{-\infty}^{\infty}|x|^{p} d \Phi(x) \mid
$$

If

$$
0<\Delta \leq \frac{1}{\sqrt{e}}
$$

then there exists a universal constant $c_{\Delta}$, depending only on $p$, such that

$$
|F(x)-\Phi(x)| \leq \frac{\lambda_{p}+c_{\Delta} \Delta\left(\ln \frac{1}{\Delta}\right)^{p / 2}}{1+|x|^{p}}
$$

for all $x \in \mathbf{R}$.

A similar result is obtained in [10 in terms of the Lévy distance instead of the uniform distance.

Theorem B (Indlekofer-Klesov). Let $F$ be an arbitrary distribution function and let $L=\mathcal{L}(F, \Phi)$. Let $p>0$ and assume that the moment of order $p$ exists for the distribution function $F$. If

$$
0<L \leq \frac{1}{\sqrt{e}}
$$

then there exists a universal constant $c_{L}$, depending only on $p$, such that

$$
|F(x)-\Phi(x)| \leq \frac{\lambda_{p}+c_{L} L\left(\ln \frac{1}{L}\right)^{p / 2}}{1+|x|^{p}}
$$

for all $x \in \mathbf{R}$, where the constant $\lambda_{p}$ is defined by (2.1).

Remark 2.1. Theorems $\mathrm{A}$ and $\mathrm{B}$ look very similar. Note, however, that in the case of

$$
L \leq \frac{1}{\sqrt{e}}<\Delta
$$

only Theorem $\mathrm{B}$ is applicable.

Remark 2.2. Since we provide a better result below, we do not go into a deep comparison of these theorems. Nevertheless a couple of comments are in order.

There are some cases in which one of the two Theorems $\mathrm{A}$ or Bimplies the other. Since the function $x\left(\ln x^{-1}\right)^{p / 2}$ increases on the interval $\left(0, e^{-p / 2}\right)$ and decreases on $\left(e^{-p / 2}, 1\right)$, assertion (2.5) implies (2.3) if

$$
\Delta \leq \min \left\{\frac{1}{\sqrt{e^{p}}}, \frac{1}{\sqrt{e}}\right\}
$$


Moreover, the constant $c_{\Delta}$ inherits the value of the constant $c_{L}$ in this case. On the other hand, (2.3) implies (2.5) if $p \geq 1$ and

$$
\frac{1}{\sqrt{e^{p}}} \leq L \leq \Delta \leq \frac{1}{\sqrt{e}}
$$

Now the constant $c_{L}$ inherits the value of the constant $c_{\Delta}$. The case of (2.6) fits better in the context of the central limit theorem compared to (2.7) (because $\Delta \rightarrow 0$ for the central limit theorem).

Since

$$
\Delta \leq\left(1+\frac{1}{\sqrt{2 \pi}}\right) L
$$

if $G=\Phi$, there are several other interrelations between Theorems $\mathrm{A}$ and $\mathrm{B}$. In what follows we use (2.5) rather than (2.3) even in the cases where both of them are applicable. This can be explained by the fact that other functions $G$ (even discontinuous ones) can be used in (2.5) in place of $\Phi$, while (2.3) has no nice generalizations in the discontinuous case if we are concerned with weak convergence. Theorem $B$ for other $G$ instead of $\Phi$ will be discussed elsewhere.

In the following result we avoid the restriction (2.4).

Theorem 1. Let $F$ be an arbitrary distribution function. Denote by $L=\mathcal{L}(F, \Phi)$ the Lévy distance between $F$ and $\Phi$. Let $p>0$ and suppose the moment of order $p$ exists for $F$. Then there exists a function $g$ defined on $(0,1)$, depending only on $p$, and such that

$$
\lim _{s \downarrow 0} g(s)=0
$$

and

$$
|F(x)-\Phi(x)| \leq \frac{\lambda_{p}+g(L)}{1+|x|^{p}}
$$

for all $x \in \mathbf{R}$, provided $0 \leq L<1$.

Remark 2.3. The case of $L=0$ is equivalent to $F=\Phi$. Indeed, for any $h \in \mathbb{H}$,

$$
\Phi(x) \geq F(x-h)-h, \quad x \in \mathbf{R} .
$$

If $L=0$, then $\Phi(x) \geq F(x), x \in \mathbf{R}$, in view of the left continuity of $F$. Exchanging $\Phi$ and $F$ in the definition of $L$, we prove the converse inequality, whence $F=\Phi$.

Remark 2.4. The case of $L=1$ is not possible for any distribution function $F$. This follows from the estimate

$$
L \leq \Delta<1, \quad \text { since } 0<\Phi(x)<1 \text { for all } x .
$$

Remark 2.5. Inequality (2.8) improves the Markov inequality in some cases. Indeed, let $F^{*}(x)$ be the tail of $F$, that is,

$$
F^{*}(x)=1-F(x)+F(-x), \quad x \geq 0 .
$$

For simplicity we assume that $p=2$ and that the first two moments of $F$ and $\Phi$ coincide. Then the Markov inequality yields

$$
F^{*}(x) \leq \frac{1}{x^{2}},
$$

while (2.8) implies that

$$
F^{*}(x) \leq\left|F^{*}(x)-\Phi^{*}(x)\right|+\Phi^{*}(x) \leq \frac{2 g(L)}{1+x^{2}}+\Phi^{*}(x) .
$$

If $L$ is small and $x$ is large, then the latter bound is better than the one from the Markov inequality. 
An explicit form of the function $g$ is available under a minor additional restriction.

Theorem 2. Let $F$ be an arbitrary distribution function and let $L=\mathcal{L}(F, \Phi)$. Let $p>0$ and assume that the moment of order $p$ exists for $F$. Finally, let $L_{0} \in(0,1)$ be fixed and $0<L \leq L_{0}$. Then there exists a constant $c_{p}$, depending only on $p$ and $L_{0}$, such that

$$
|F(x)-\Phi(x)| \leq \frac{\lambda_{p}+c_{p} L\left(\ln \frac{1}{L}\right)^{p / 2}}{1+|x|^{p}}
$$

for all $x \in \mathbf{R}$.

Theorem 2 coincides with Theorem $\mathrm{B}$ if $L_{0}=e^{-1 / 2}$. Note also that the constant $c_{p}$ decreases with $L_{0}$ if $p$ is fixed.

Proof of Theorem 1. In view of Remarks 2.3 and 2.4, we restrict our consideration to the case of $0<L<1$. The proof below uses the main ideas of the proof in [11].

Denote by $\mathcal{C}(F)$ the set of continuity points of $F$. For all $a>0$ such that $\pm a \in \mathcal{C}(F)$ we have

$$
\begin{aligned}
& \int_{(-a, a)}|x|^{p} d F(x) \\
& =\int_{(-a, a)}|x|^{p} d[F(x)-\Phi(x)]+\int_{(-a, a)}|x|^{p} d \Phi(x) \\
& =a^{p}[F(a)-\Phi(a)]-a^{p}[F(-a)-\Phi(-a)] \\
& -p \int_{(0, a)} x^{p-1}[F(x)-\Phi(x)] d x \\
& +p \int_{(-a, 0)}|x|^{p-1}[F(x)-\Phi(x)] d x+\int_{(-a, a)}|x|^{p} d \Phi(x) .
\end{aligned}
$$

For all $h \in \mathbb{H}(F, \Phi)$ and all $x \in \mathbf{R}$,

$$
\begin{aligned}
F(x)-\Phi(x) & =F(x)-\Phi(x-h)+h-h+\Phi(x-h)-\Phi(x) \\
& \geq-h-[\Phi(x)-\Phi(x-h)] .
\end{aligned}
$$

By the mean value theorem and the fact that $\Phi^{\prime}(\xi)=\varphi(\xi) \leq 1 / \sqrt{2 \pi}$,

$$
\Phi(x)-\Phi(x-h) \leq \frac{h}{\sqrt{2 \pi}},
$$

whence

$$
F(x)-\Phi(x) \geq-h\left(1+\frac{1}{\sqrt{2 \pi}}\right), \quad h \in \mathbb{H}(F, \Phi),
$$

for all $x \in \mathbf{R}$. In particular,

$$
F(a)-\Phi(a) \geq-h\left(1+\frac{1}{\sqrt{2 \pi}}\right), \quad h \in \mathbb{H}(F, \Phi) .
$$

Similarly,

$$
\begin{aligned}
F(x)-\Phi(x) & =F(x)-\Phi(x+h)-h+h+\Phi(x+h)-\Phi(x) \\
& \leq h+[\Phi(x+h)-\Phi(x)]
\end{aligned}
$$

and thus

$$
F(x)-\Phi(x) \leq h\left(1+\frac{1}{\sqrt{2 \pi}}\right), \quad h \in \mathbb{H}(F, \Phi),
$$


for all $x \in \mathbf{R}$. In particular,

$$
F(-a)-\Phi(-a) \leq h\left(1+\frac{1}{\sqrt{2 \pi}}\right), \quad h \in \mathbb{H}(F, \Phi) .
$$

Applying (2.14) we obtain, for every $h \in \mathbb{H}(F, \Phi)$, that

$$
\begin{aligned}
\int_{(0, a)}|x|^{p-1}[F(x)-\Phi(x)] d x & \leq \int_{(0, a)}|x|^{p-1} h\left(1+\frac{1}{\sqrt{2 \pi}}\right) d x \\
& =\frac{1}{p} a^{p} h\left(1+\frac{1}{\sqrt{2 \pi}}\right) .
\end{aligned}
$$

Similarly, from (2.12), we derive that

$$
\int_{(-a, 0)}|x|^{p-1}[F(x)-\Phi(x)] d x \geq-\frac{1}{p} a^{p} h\left(1+\frac{1}{\sqrt{2 \pi}}\right) .
$$

Combining the latter estimates with (2.13), (2.15) and inserting them into (2.11), we obtain

$$
\int_{(-a, a)}|x|^{p} d F(x) \geq-h B_{p}+\int_{(-a, a)}|x|^{p} d \Phi(x),
$$

where

$$
B_{p}=4\left(1+\frac{1}{\sqrt{2 \pi}}\right) a^{p} .
$$

Recalling the definition of $\lambda_{p}$, we have

$$
\begin{aligned}
\lambda_{p} \geq & \int_{|x|<a}|x|^{p} d F(x)-\int_{|x|<a}|x|^{p} d \Phi(x) \\
& +\int_{|x| \geq a}|x|^{p} d F(x)-\int_{|x| \geq a}|x|^{p} d \Phi(x) \\
\geq & -h B_{p}+\int_{|x| \geq a}|x|^{p} d F(x)-\int_{x \geq a}|x|^{p} d \Phi(x),
\end{aligned}
$$

whence

$$
\int_{|x| \geq a}|x|^{p} d F(x) \leq \lambda_{p}+h B_{p}+\int_{|x| \geq a}|x|^{p} d \Phi(x) .
$$

Further, if $x \geq a$, then

$$
\int_{|y| \geq a}|y|^{p} d F(y) \geq \int_{y \geq x} y^{p} d F(y) \geq x^{p}(1-F(x)) \geq x^{p}[\Phi(x)-F(x)] .
$$

Therefore, for every $h \in \mathbb{H}(F, \Phi)$,

$$
x^{p}[\Phi(x)-F(x)] \leq \lambda_{p}+h B_{p}+\int_{|y| \geq a}|y|^{p} d \Phi(y) .
$$

Now,

$$
\begin{aligned}
F(x) & -\Phi(x) \leq 1-\Phi(x) \\
& \leq \int_{|y| \geq x} d \Phi(y) \leq \frac{1}{x^{p}} \int_{|y| \geq x}|y|^{p} d \Phi(y), \quad x \in \mathbf{R},
\end{aligned}
$$

whence, for all $x \geq a$,

$$
x^{p}[F(x)-\Phi(x)] \leq \int_{|y| \geq a}|y|^{p} d \Phi(y)
$$


Combining this bound with (2.17), we get, for $x \geq a$ and $h \in \mathbb{H}(F, \Phi)$, that

$$
|x|^{p}|F(x)-\Phi(x)| \leq \lambda_{p}+h B_{p}+\int_{|y| \geq a}|y|^{p} d \Phi(y) .
$$

A similar bound holds for $x \leq-a$. Finally, in view of (2.12) and (2.14), it also holds for $|x|<a$. Therefore (2.19) holds for all $x \in \mathbf{R}$.

The same reasoning applies for $p=0$. Note that $\lambda_{0}=0$. Thus

$$
\begin{aligned}
& \left(1+|x|^{p}\right)|F(x)-\Phi(x)| \\
& \quad \leq \lambda_{p}+h B_{p}+h B_{0}+\int_{|y| \geq a}|y|^{p} d \Phi(y)+\int_{|y| \geq a} d \Phi(y) .
\end{aligned}
$$

The right-hand side of this estimate is a continuous function of $a$ (see (2.16) $)$; therefore, one can remove the assumption that $\pm a \in \mathcal{C}(F)$. Thus (2.20) holds for all $a>0$. Moreover, on taking the infimum over $h \in \mathbb{H}(F, \Phi)$, we have, for all $x \in \mathbf{R}$ and all $a>0$, that

$$
\begin{aligned}
& \left(1+|x|^{p}\right)|F(x)-\Phi(x)| \\
& \quad \leq \lambda_{p}+L B_{p}+L B_{0}+\int_{|y| \geq a}|y|^{p} d \Phi(y)+\int_{|y| \geq a} d \Phi(y),
\end{aligned}
$$

where $L$ is the Lévy distance between $F$ and $\Phi$. Thus (2.8) follows with

$$
g(L)=\inf _{a>0} f(L, a)
$$

where, for $0 \leq s<1$ and $a>0$,

$$
f(s, a)=s \kappa a^{p}+s \kappa+\int_{|y| \geq a}|y|^{p} \varphi(y) d y+\int_{|y| \geq a} \varphi(y) d y,
$$

with $\kappa=4(1+1 / \sqrt{2 \pi})$ (see (2.16) $)$.

If $\alpha$ is an increasing function on $(1, \infty)$ such that

$$
\lim _{z \rightarrow \infty} \alpha(z)=\infty, \quad \lim _{z \rightarrow \infty} \frac{\alpha(z)}{z^{1 / p}}=0,
$$

then

$$
g(s) \leq f(s, \alpha(1 / s)) \rightarrow 0, \quad s \downarrow 0 .
$$

This completes the proof of Theorem 1

Proof of Theorem 2, First we proceed as in the proof of Theorem 1 and arrive at (2.21). We choose $\alpha(z)=\sqrt{2 \ln z}$ and consider the function in (2.22) for $a \geq a_{0}$, where

$$
a=\sqrt{2 \ln \frac{1}{L}} \text { and } a_{0}=\sqrt{2 \ln \frac{1}{L_{0}}} .
$$

Then

and

$$
L \leq c_{1} L\left(\ln \frac{1}{L}\right)^{p / 2}, \quad \text { with } \quad c_{1}=\left(\ln \frac{1}{L_{0}}\right)^{-p / 2}
$$

$$
\int_{a}^{\infty} \varphi(x) d x \leq c_{2} \int_{a}^{\infty} x^{p} \varphi(x) d x, \quad \text { with } c_{2}=a_{0}^{-p} .
$$

Note also that, for some constant $c_{3}$ depending on $a_{0}$,

$$
\int_{a}^{\infty} x^{p} \varphi(x) d x \leq c_{3} a^{p-1} \varphi(a), \quad a \geq a_{0} .
$$


Thus

$$
\begin{aligned}
f(L, a) & \leq c_{3}\left(L a^{p}+\int_{a}^{\infty} x^{p} \varphi(x) d x\right) \\
& \leq c_{4}\left(L\left(\ln \frac{1}{L}\right)^{p / 2}+L\left(\ln \frac{1}{L}\right)^{(p-1) / 2}\right) \\
& \leq c_{5} L\left(\ln \frac{1}{L}\right)^{p / 2} .
\end{aligned}
$$

\section{Generalized global version of the Central limit theorem}

Agnew 2] was apparently the first to study the relationship between weak convergence and the convergence to zero of the integral in (3.3) below. We should mention, however, that in Agnew's setting the limit distribution function can be arbitrary and not just $\Phi$. Nevertheless, we restrict our considerations here to $\Phi$ as a weak limit in order to highlight the main features of our approach. Moreover, for the sake of comparison, we state the results of other authors also with $\Phi$ only, even if they have been proved for more general limiting distribution functions.

Similar results can also be obtained via Theorem $\mathrm{A}$ (see, e.g., [15] and [16]). Another method of proof is due to Studnev and Ignat [18] (see also [12]).

For example, from Agnew [2] we have the following result.

Theorem C (Agnew [2]). Let $\left\{F_{n}\right\}$ be a sequence of distribution functions such that

$$
\int_{-\infty}^{\infty} x d F_{n}(x)=0 \quad \text { and } \quad \int_{-\infty}^{\infty} x^{2} d F_{n}(x)=1
$$

Suppose

$$
F_{n} \stackrel{w}{\longrightarrow} \Phi, \quad n \rightarrow \infty .
$$

Then, for all $r>\frac{1}{2}$,

$$
\int_{-\infty}^{\infty}\left|F_{n}(x)-\Phi(x)\right|^{r} d x \rightarrow 0, \quad n \rightarrow \infty .
$$

In fact, Agnew [2] proved that the weak convergence is equivalent to (3.3) in a certain sense.

We can complete this result by considering also the case of $r=\frac{1}{2}$.

Theorem 3. Let $\left\{F_{n}\right\}$ be a sequence of distribution functions satisfying (3.1). If (3.2) holds, then

$$
\int_{-\infty}^{\infty} \frac{\left|F_{n}(x)-\Phi(x)\right|^{1 / 2}}{(\ln (1+|x|))^{1+\delta}} d x \rightarrow 0, \quad n \rightarrow \infty
$$

for all $\delta>0$.

Note that the distribution functions $\left\{F_{n}\right\}$ in Theorem $[\mathrm{C}$ are arbitrary and need not correspond to partial sums of independent random variables. The latter more specific and more typical case will be considered next.

Corollary 1. Let $\left\{X_{n}\right\}$ be a sequence of independent, identically distributed random variables and let $\left\{S_{n}\right\}$ denote their partial sums. Assume that

$$
\mathrm{E} X_{1}=0 \text { and } \mathrm{E} X_{1}^{2}=1 \text {. }
$$

If $r>\frac{1}{2}$, then (3.3) holds, where $F_{n}$ denotes the distribution function of $n^{-1 / 2} S_{n}$. 
This result was generalized by Esseen [6] to the case of independent, but not necessarily identically distributed random variables $\left\{X_{n}\right\}$, provided they satisfy the central limit theorem. A further extension is due to de Acosta and Giné [1].

Of course, the smaller the value of $r>0$, the stronger is the convergence in (3.3). So, it is natural to ask whether the restriction $r>\frac{1}{2}$ in the above results can be weakened to $r>0$. A first positive answer has been given by Nishimura 14. However, as pointed out in Rosalsky [17, the argument in [14] is incomplete and even the assumptions can be improved.

Theorem D (Laube [13] and Rosalsky [17]). Let $\left\{F_{n}\right\}$ be a sequence of distribution functions such that

$$
\sup _{n \geq 1} \int_{-\infty}^{\infty}|x|^{p} d F_{n}(x)<\infty
$$

for some $p>1$. Assume that $F_{n}$ converges weakly to $\Phi$ as $n \rightarrow \infty$. Then (3.3) holds for all $r>1 / p$.

We extend Theorem $\mathrm{D}$ to the case $r=1 / p$.

Theorem 4. Suppose all the assumptions of Theorem $\mathrm{D}$ hold. Then

for all $\delta>0$.

$$
\int_{-\infty}^{\infty} \frac{\left|F_{n}(x)-\Phi(x)\right|^{1 / p}}{(\ln (1+|x|))^{1+\delta}} d x \rightarrow 0, \quad n \rightarrow \infty
$$

The proof of Theorem 4 is the same as that of Theorem 3.

Theorem D can be generalized as follows.

Theorem 5. Let $p>0$ and $r>0$. Let $\left\{F_{n}\right\}$ be a sequence of distribution functions such that $F_{n} \stackrel{w}{\longrightarrow} \Phi(n \rightarrow \infty)$ and condition (3.5) holds. Let $g$ be a positive function such that

$$
\int_{-\infty}^{\infty} \frac{g(x)}{(1+|x|)^{r p_{1}}} d x<\infty \text { for some } 0<p_{1}<p .
$$

Then

$$
\int_{-\infty}^{\infty} g(x)\left|F_{n}(x)-\Phi(x)\right|^{r} d x \rightarrow 0, \quad n \rightarrow \infty .
$$

Remark 3.1. The case of $g(x)=1+|x|^{q}, q \geq 0$, in Theorem [5] has been proved by V. V. Petrov (see Theorem 14 in Chapter 1 of [16]). For this case condition (3.7) reduces to $p r>1+q$.

The case of $q=0$ in Petrov's theorem corresponds to Theorem D. For the particular case of $q=0, p=2$, Petrov shows that the result can be proved via Theorem $\mathrm{A}$ (see Theorem 15, $\S 5$, Chapter V of [15] or $\S 5$, Chapter V of [16]).

Excluding the weak convergence from the set of assumptions in Theorem 5. we obtain the following general result.

Theorem 6. Let $p>0$ and $r>0$. Let $\left\{F_{n}\right\}$ be a sequence of distribution functions such that condition (3.5) holds. Let $g$ be a positive function satisfying (3.7). Then

$$
\limsup _{n \rightarrow \infty} \int_{-\infty}^{\infty} g(x)\left|F_{n}(x)-\Phi(x)\right|^{r} d x<\infty .
$$

Remark 3.2. In the proofs below, we apply Theorem 2, One of the assumptions of this theorem concerns the upper bound $L_{0}$ for the Lévy distances. Thus we must have

$$
\limsup _{n \rightarrow \infty} L_{n}<1
$$


in order to apply Theorem 2. We derive relation (3.8) from the boundedness of moments required in (3.5). Indeed, denote $L_{n}=\mathcal{L}\left(F_{n}, \Phi\right)$ and $\Delta_{n}=\Delta\left(F_{n}, \Phi\right)$. If (3.8) does not hold, then, for some subsequence $\left\{n_{k}\right\}$,

$$
\lim _{k \rightarrow \infty} L_{n_{k}}=1 \text {, }
$$

whence

$$
\lim _{k \rightarrow \infty} \Delta_{n_{k}}=1 .
$$

Without loss of generality we assume that $\left\{n_{k}\right\}$ coincides with the whole sequence of integers. Then, there is a subsequence $\left\{x_{n}\right\}$ such that

$$
\left|F_{n}\left(x_{n}\right)-\Phi\left(x_{n}\right)\right| \rightarrow 1 \text {. }
$$

Choose a convergent subsequence of $\left\{\Phi\left(x_{n}\right)\right\}$ and assume again without loss of generality that it coincides with the whole sequence of integers. Obviously it converges either to 0 or to 1 . In the first case, $x_{n} \rightarrow-\infty$ and $F_{n}\left(x_{n}\right) \rightarrow 1$, while, in the second case, $x_{n} \rightarrow \infty$ and $F_{n}\left(x_{n}\right) \rightarrow 0$. In either case, $F_{n}^{*}(c) \rightarrow 1$ for all $c>0$ (see (2.9) for the definition of the tail of a distribution function). Thus, for any $c>0$,

$$
\int_{-\infty}^{\infty}|x|^{p} d F_{n} \geq \int_{|x| \geq c}|x|^{p} d F_{n} \geq c^{p} F_{n}^{*}(c)
$$

which clearly contradicts (3.5), since $c$ is arbitrary.

Proof of Theorem 3, In view of Remark 3.2, there is an $L_{0}<1$ such that $L_{n} \leq L_{0}$ for all $n \geq 1$. Then, by Theorem 2, for $p=2$ and with $\lambda_{p}=0$,

$$
\begin{aligned}
\int_{-\infty}^{\infty} \frac{\left|F_{n}(x)-\Phi(x)\right|^{1 / 2}}{(\ln (1+|x|))^{1+\delta}} d x & \leq \int_{-\infty}^{\infty}\left(\frac{c_{2} L_{n} \ln \frac{1}{L_{n}}}{1+x^{2}}\right)^{1 / 2} \frac{1}{(\ln (1+|x|))^{1+\delta}} d x \\
& =o(1) \int_{-\infty}^{\infty} \frac{1}{\left(1+x^{2}\right)^{1 / 2}(\ln (1+|x|))^{1+\delta}} d x
\end{aligned}
$$

This estimate proves Theorem 3 .

Proof of Theorem 5, In view of Remark 3.2, there is an $L_{0}<1$ such that $L_{n} \leq L_{0}$ for all $n \geq 1$. Then, by Theorem 2 ,

$$
\begin{aligned}
\int_{-\infty}^{\infty} g(x)\left|F_{n}(x)-\Phi(x)\right|^{r} d x & \leq \int_{-\infty}^{\infty} g(x)\left(\frac{\lambda_{p_{1}, n}+c_{p_{1}} L_{n}\left(\ln \frac{1}{L_{n}}\right)^{p_{1} / 2}}{(1+|x|)^{p_{1}}}\right)^{r} d x \\
& =o(1) \int_{-\infty}^{\infty} \frac{g(x)}{(1+|x|)^{r p_{1}}} d x,
\end{aligned}
$$

where $o(1) \rightarrow 0$ as $n \rightarrow \infty$. This completes the proof of Theorem 5

Proof of Theorem 6. Here one can proceed in the same way as in the proof of Theorem 5. The only difference is that $O(1)$ replaces $o(1)$.

\section{Generalized complete convergence}

The concept of complete convergence has been introduced by Hsu and Robbins in [9]. By definition, a sequence of random variables $\left\{\xi_{n}\right\}$ is said to converge completely to zero (denoted by $\xi_{n} \stackrel{\text { c.c. }}{\longrightarrow} 0, n \rightarrow \infty$ ) if not only $\xi_{n} \stackrel{\text { a.s. }}{\longrightarrow} 0$ but also $\xi_{n}^{\prime} \stackrel{\text { a.s. }}{\longrightarrow} 0$ as $n \rightarrow \infty$, for any sequence of random variables $\left\{\xi_{n}^{\prime}\right\}$ such that $\xi_{n} \stackrel{\mathrm{d}}{=} \xi_{n}^{\prime}$ for all $n \geq 1$, i.e., for $\xi_{n}$ and $\xi_{n}^{\prime}$ having the same distribution function for all $n \geq 1$. Note that other limit values are easily obtained from this case. 
Remark 4.1. Hsu and Robbins [9] proved that the complete convergence $\xi_{n} \stackrel{\text { c.c. }}{\longrightarrow} 0$ as $n \rightarrow \infty$ is equivalent to

$$
\sum_{n=1}^{\infty} \mathrm{P}\left(\left|\xi_{n}\right| \geq \varepsilon\right)<\infty \text { for all } \varepsilon>0
$$

Using the notation $F^{*}(x)$ for the tail of a distribution function $F$ (cf. (2.9)), we see that the latter condition is equivalent to

$$
\sum_{n=1}^{\infty} F_{n}^{*}(\varepsilon)<\infty \text { for all } \varepsilon>0
$$

where $F_{n}$ denotes the distribution function of $\xi_{n}$.

The convergence $n^{-1} S_{n} \stackrel{\text { c.c. }}{\longrightarrow} 0$ as $n \rightarrow \infty$ has been studied by Hsu and Robbins [9] for the case where $\left\{S_{n}\right\}$ are partial sums of independent, identically distributed random variables $\left\{X_{n}\right\}$.

Theorem E (Hsu and Robbins [9]). Let $\left\{X_{n}\right\}$ be a sequence of independent, identically distributed random variables and let $\left\{S_{n}\right\}$ denote their partial sums. Then $n^{-1} S_{n} \stackrel{\text { c.c. }}{\longrightarrow} 0$ as $n \rightarrow \infty$ if and only if condition (3.1) holds.

If $F_{n}$ denotes the distribution function of $n^{-1 / 2} S_{n}$ (with corresponding $F_{n}^{*}$ ), Theorem E can be stated as follows:

$$
\frac{S_{n}}{n} \stackrel{\text { c.c. }}{\longrightarrow} 0, n \rightarrow \infty \quad \Longleftrightarrow \quad \text { (3.1) holds } \Longleftrightarrow \sum_{n=1}^{\infty} F_{n}^{*}(\varepsilon \sqrt{n})<\infty \text { for all } \varepsilon>0 \text {. }
$$

The following result provides conditions for the convergence of the latter series in the general case.

Theorem 7. Let $\left\{F_{n}\right\}$ be an arbitrary sequence of distribution functions. Assume that condition (3.5) holds with some $p>2$. Then

$$
\sum_{n=1}^{\infty} F_{n}^{*}(\varepsilon \sqrt{n})<\infty \text { for all } \varepsilon>0 .
$$

We do not give the proof of Theorem [7 since it follows the lines of the proof of more general Theorem 8 below.

Heyde [8] considered the asymptotics as $\varepsilon \downarrow 0$ of the series (4.1) in the Hsu-Robbins case of sums of independent, identically distributed random variables.

Theorem F (Heyde [8]). Let $\left\{X_{n}\right\}$ be a sequence of independent, identically distributed random variables and let $\left\{S_{n}\right\}$ denote their partial sums. Let $F_{n}$ be the distribution function of $n^{-1 / 2} S_{n}$ and let $F_{n}^{*}$ be the tail of $F_{n}$. If condition (3.1) holds, then

$$
\lim _{\varepsilon \downarrow 0} \varepsilon^{2} \sum_{n=1}^{\infty} F_{n}^{*}(\varepsilon \sqrt{n})=1
$$

We complete this result as follows.

Theorem 8. Let $p>2$. Let $\left\{F_{n}\right\}$ be a sequence of distribution functions such that $F_{n} \stackrel{w}{\longrightarrow} \mathcal{N}\left(0, \sigma^{2}\right), \sigma^{2}>0$, and condition (3.5) holds. Then

$$
\lim _{\varepsilon \downarrow 0} \varepsilon^{2} \sum_{n=1}^{\infty} F_{n}^{*}(\varepsilon \sqrt{n})=\sigma^{2}
$$


Proof. We prove the theorem for the case of $\sigma^{2}=1$. The general case of $\sigma^{2}$ immediately follows from this special case. Therefore, we assume that $F_{n} \stackrel{w}{\longrightarrow} \Phi$.

First we note that

$$
\lim _{\varepsilon \downarrow 0} \varepsilon^{2} \sum_{n=1}^{\infty} \Phi^{*}(\varepsilon \sqrt{n})=1
$$

by Theorem $\mathrm{F}$ for Gaussian random variables $X_{n}$. Further let $c>0$ be fixed. Then

$$
\sum_{n<c \varepsilon^{-2}}\left|F_{n}^{*}(\varepsilon \sqrt{n})-\Phi^{*}(\varepsilon \sqrt{n})\right| \leq \frac{c}{\varepsilon^{2}},
$$

whence

$$
\limsup _{\varepsilon \downarrow 0} \varepsilon^{2} \sum_{n<c \varepsilon^{-2}}\left|F_{n}^{*}(\varepsilon \sqrt{n})-\Phi^{*}(\varepsilon \sqrt{n})\right| \leq c .
$$

Now let $2<p_{1}<p$. In view of Remark 3.2 , there is an $L_{0}<1$ such that $L_{n} \leq L_{0}$ for all $n \geq 1$. Then, by Theorem 2 ,

$$
\sum_{n \geq c \varepsilon^{-2}}\left|F_{n}^{*}(\varepsilon \sqrt{n})-\Phi^{*}(\varepsilon \sqrt{n})\right| \leq 2 \sum_{n \geq c \varepsilon^{-2}} \frac{\lambda_{p_{1}, n}+c_{p_{1}} L_{n}\left(\ln \frac{1}{L_{n}}\right)^{p_{1} / 2}}{(1+\varepsilon \sqrt{n})^{p_{1}}},
$$

whence

$$
\sum_{n \geq c \varepsilon^{-2}}\left|F_{n}^{*}(\varepsilon \sqrt{n})-\Phi^{*}(\varepsilon \sqrt{n})\right|=o(1) \sum_{n \geq c \varepsilon^{-2}} \frac{1}{(1+\varepsilon \sqrt{n})^{p_{1}}}
$$

where $o(1) \rightarrow 0$ as $\varepsilon \downarrow 0$. Therefore

$$
\varepsilon^{2} \sum_{n \geq c \varepsilon^{-2}}\left|F_{n}^{*}(\varepsilon \sqrt{n})-\Phi^{*}(\varepsilon \sqrt{n})\right|=o(1) .
$$

This together with (4.5) gives

$$
\limsup _{\varepsilon \downarrow 0} \varepsilon^{2} \sum_{n=1}^{\infty}\left|F_{n}^{*}(\varepsilon \sqrt{n})-\Phi^{*}(\varepsilon \sqrt{n})\right| \leq c .
$$

Since $c>0$ is arbitrary, (4.3) can be derived from (4.4).

\section{BIBLIOGRAPHY}

1. A. de Acosta and E. Giné, Convergence of moments and related functionals in the central limit theorem in Banach spaces, Z. Wahrsch. Verw. Gebiete 48 (1979), 213-231. MR.534846 (80h:60011)

2. R. P. Agnew, Global versions of the central limit theorem, Proc. Nat. Acad. Sci. U.S.A. 48 (1954), 800-804. MR0064342(16:268d)

3. R. P. Agnew, Estimates for global central limit theorems, Ann. Math. Statist. 28 (1957), 26-42. MR 0084227 (18:832f)

4. R. P. Agnew, Asymptotic expansions in global central limit theorems, Ann. Math. Statist. 30 (1959), 721-737. MR0107919 (21:6641)

5. C. G. Esseen, Fourier analysis of distribution functions. A mathematical study of the LaplaceGaussian law, Acta Math. 77 (1945), 1-125. MR0014626 (7:312a)

6. C. G. Esseen, On mean central limit theorems, Kungl. Tekn. Högsk. Handl. Stockholm 121 (1958), 1-31. MR0097111 (20:3591)

7. B. V. Gnedenko and A. N. Kolmogorov, Limit Distributions for Sums of Independent Random Variables, GTTI, Moscow-Leningrad, 1949; English transl., Cambridge, Addison-Wesley, 1954. MR0062975 (16:52d)

8. C. C. Heyde, A supplement to the strong law of large numbers, J. Appl. Probab. 12 (1975), 173-175. MR0368116 (51:4358)

9. P. L. Hsu and H. Robbins, Complete convergence and the law of large numbers, Proc. Nat. Acad. Sci. U.S.A. 33 (1947), 25-31. MR0019852 (8:470e) 
10. K.-H. Indlekofer and O. I. Klesov, A generalization of a Kolodyazhny̌ theorem for the Lévy distance, Internat. J. Pure Appl. Math. 47 (2008), 235-241. MR.2457827 (2009k:60063)

11. A. F. Kolodyazhnyı̆, A generalization of a theorem of Esseen, Vestnik. Leningrad. Univ. 13 (1968), 28-33. (Russian)

12. V. M. Kruglov, Convergence of numeric characteristics of sums of independent random variables and global theorems, Lecture Notes in Math., Proceedings of the Second Japan-USSR Symposium on Probability Theory, vol. 330, Springer-Verlag, New York-Heidelberg-Berlin, 1973, pp. 255-286. MR0445582 (56:3919)

13. G. Laube, Weak convergence and convergence in the mean of distribution functions, Metrika 20 (1973), 103-105. MR0407939 (53:11706)

14. S. Nishimura, An inequality for a metric in a random collision process, J. Appl. Prob. 12 (1975), 239-247. MR0381040 (52:1937)

15. V. V. Petrov, Sums of Independent Random Variables, Nauka, Moscow, 1972; English transl., Springer-Verlag, New York-Heidelberg-Berlin, 1975. MR0322927 (48:1288)

16. V. V. Petrov, Limit Theorems of Probability Theory. Sequences of Independent Random Variables, Nauka, Moscow, 1987; English transl., Oxford University Press, Oxford, 1995. MR.1353441 (96h:60048)

17. A. Rosalsky, A generalization of the global limit theorems of R. P. Agnew, Internat. J. Math. \& Math. Sci. 11 (1988), 365-374. MR.939092 (89f:60028a)

18. Yu. P. Studnev and Yu. I. Ignat, Refinement of the central limit theorem and of its global version, Teor. Veroyatnost. i Primenen. 12 (1967), 562-567; English transl. in Theory Probab. Appl. 12 (1967), 508-512. MR0215348 (35:6189)

Fakultät für Elektrotechnik, Informatik und Mathematik, Institut für Mathematik, Universität Paderborn, Warburger Strasse 100, Paderborn 33098, Germany

E-mail address: k-heinz@uni-paderborn.de

Department of Mathematical Analysis and Probability Theory, National Technical University of Ukraine (KPI), Prospekt Peremogy 37, Kyiv 03056, Ukraine

E-mail address: oleg@math.uni-paderborn.de

Mathematisches Institut, Universität Zu Köln, Weyertal 86-90, Köln D-50931, Germany

E-mail address: jost@math.uni-koeln.de

Received 11/SEP/2009

Translated by THE AUTHORS 